\title{
Tibetische Meister und ihr »befreites Wirken«
}

\author{
Auf Spurensuche monastischer Gemeinschaften \\ in den Lebensgeschichten des mittelalterlichen Tibet
}

Von Mathias Fermer

\author{
Janyang Dragpä Pel, \\ Verkörperung aller Buddhas, \\ den böchsten Zustand von Buddhaschaft \\ (Vajrasattva) erlangt \\ erscheinst $[\mathrm{Du}]$ dennoch als Lebrer \\ für das Wobl der fiiblenden Wesen. \\ [Dein] befreites Wirken will ich \\ zusammenfassend schildern. ${ }^{1}$
}

\section{Monastische Gemeinschaften (gompa)}

Enthaltsames Leben im Kontext monastischer Gemeinschaften war in weiten Teilen Tibets spätestens seit der Epoche, die aus europäischer Sicht als Spätmittelalter bezeichnet wird, ein charakteristischer Aspekt des religiösen Zusammenlebens. Basierend auf den im frühen Buddhismus verkündeten Idealen der »Hauslosigkeit« und Weltabkehr hat es sich in seiner traditionellen Form bis zur chinesischen Annektierung in der Mitte des 20. Jahrhunderts auf der tibetischen Hochebene erhalten.

Parallel dazu existierte in Tibet fortwährend eine außermonastische Laientradition religiöser Spezialisten, die als lokale Ritualexperten oder umherziehende Yogis und Asketen auftraten und die Einhaltung monastischer Gelübde und das institutionalisierte Klosterleben als nicht grundlegend für den spirituellen Heilserwerb erachteten.

Nähere quantitative Daten zu Größe und regionaler Verbreitung von Klöstern (gompa) lassen sich auf Basis einheimischer Quellen religiöser Geschichtsschreibung für die Periode der sogenannten Hegemonialzeit (11. bis 16. Jahrhundert) nur schwer erheben. Die erste umfassendere und gebietsübergreifende Dokumentation über Klosterinstitutionen und deren Insassen findet sich in der 1694 abgefaßten Ordensgeschichte der Gelugpa-Lehrtradition. Desi Sangye Gyatso (1653-1705), Regent des 5. Dalai Lama, berechnet hier für die kulturellen und politischen Kernregionen des Hochlandes (ohne die Region Amdo) eine Zahl von 1807 Klöstern mit einer Gesamtanzahl von 97538 Mönchen verschiedener Ordenszugehörigkeit. $^{2}$

Für das vormoderne Tỉbet in den fünfziger Jahren des 20. Jahrhunderts existieren grobe Schätzungen von Seiten chinesischer Regierungsstellen und der tibetischen Exilregierung in Dharamsala (Indien), die gleichermaßen zu dem Ergebnis kommen, daß 20 bis 30 Prozent der männlichen Bevölkerung Tibets zu jener Zeit als Mönche in klösterlichen Institutionen lebten. ${ }^{3}$ Mit einem Fünftel des Bevölkerungsanteils liegt die Zahl ordinierter Mönche damit verhältnismäßig hoch für eine buddhistisch geprägte Gesellschaft. Da bis heute keine systematische Erhebung über vormals existente Klostersiedlungen und die Zahl ihrer Bewohner stattfinden hat können, ist allerdings anzunehmen, daß es sich bei diesen Kalkulationen um vorläufige Hochrechnungen handelt. Seit den Wirren der Kulturrevolution ist der Großteil der religiösen Stätten, Klöster, Tempel und Einsiedeleien, nicht mehr existent und geographisch schwer zu lokalisieren. Zugleich leben heutzutage nur noch wenige Klosterinsassen aus der Zeit vor dem Einmarsch chinesischer Truppen als mögliche Informanten für eine retrospektive Bestandsaufnahme. Gleichwohl aber deutet das bis in die fünfziger Jahre weit verbreitete Mönchstum auf die gesellschaftliche Bedeutung, welche Klöster vormals gespielt haben.

In ähnlicher Weise zeichnen die Quellen des 14. und 15. Jahrhunderts das Bild eines stark ausgeprägten Klosterwesens, welches sich im Zuge der »Späten Ausbreitung« [des Buddhismus] (ab dem 11. Jahrhundert) als die bevorzugte Form professionell-religiöser Lebensführung in großen Teilen Tibets durchgesetzt zu haben scheint. Tibets erstes Kloster Samye wurde in der zweiten Hälfte des 8. Jahrhunderts während der Regierungszeit von Trisong Detsen (754-797), des Monarchen, der die Bekehrung Tibets initiierte, erbaut, als die neuimportierte Heilslehre aus Indien und Zentralasien per Dekret zur gültigen Staatsreligion erklärt wurde. Der Zerfall des tibetischen Großreichs im 9. Jahrhundert führte kurz später jedoch zum Niedergang der monastischen Tradition und beendete die von tibetischen Historikern in glorreicher Erinnerung gehaltene Epoche der sogenannten »Frühen Ausbreitung« [des Buddhismus].

Mit dem Wiederaufleben des Buddhismus im späten 10. Jahrhundert entstand eine Vielzahl neuer Klöster und Ordensschulen im Umfeld charismatischer Akteure, welche sich in buddhistischer Sittlichkeit, Ritualaktivität und kontemplativer Übung schulten. Jene neuen Gruppierungen folgten hierbei verschiedenen Lehrtraditionen des indischen Buddhismus, welcher sich, bedroht von muslimischen Invasoren im Süden, bis auf das tibetische Hochland flüchtete. Ungeachtet der Unterschiede im Hinblick auf monastische Verrichtungen und doktrinäre Deutungsspielräume ist den Klostergemeinschaften gemein, daß sie ihren Ursprung in indischen Meistern und deren Lehrverkündung sehen. Die in Tibet zu Ansehen gelangten Meister und ihre Gefolgschaft waren wirtschaftlich auf Zuwendungen aus den 


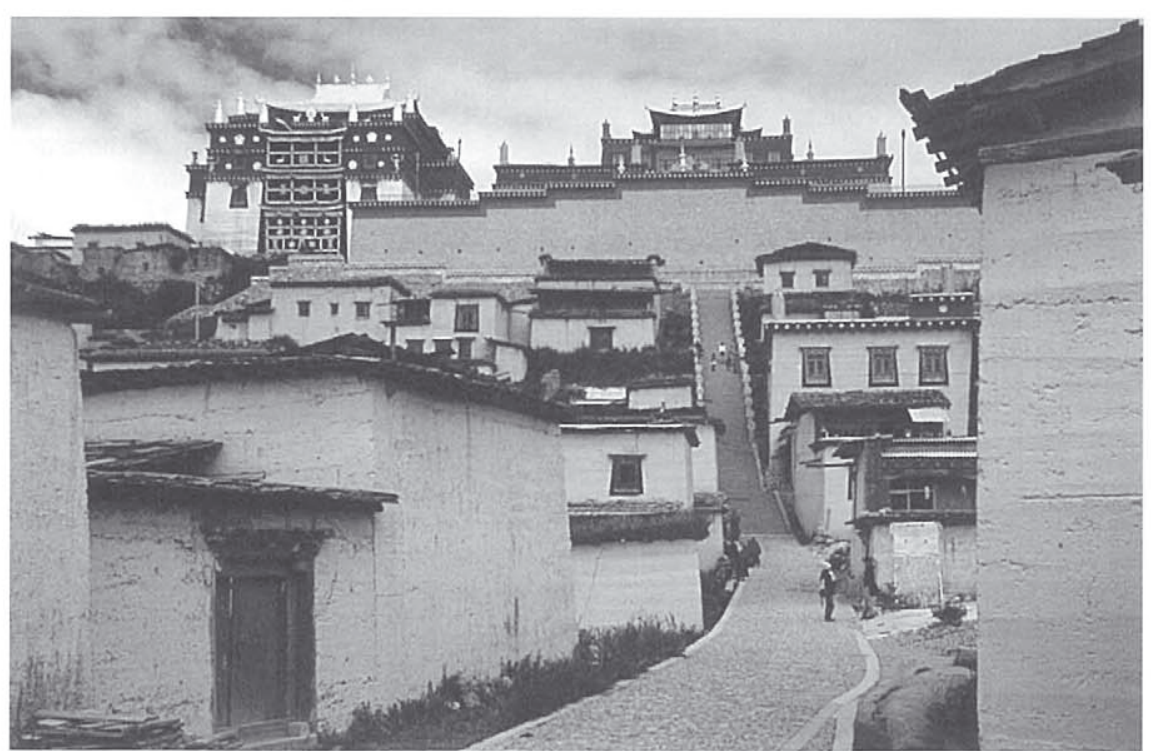

Reihen regionaler Machthaber und der ansässigen Bevölkerung angewiesen. So traten sie als sachkundige Lehrmeister und Ritualexperten mit lokalen Klangruppen in ein wechselseitiges »Lehrer-Gabenherr»-Verhältnis. Einflußreiche Klanfamilien, welche territorialen Raum in ihren Besitz gebracht hatten und ihren Herrschaftsanspruch auf anzestrale Linien aus der Königszeit zurückführten, förderten das Wirken geistlicher Lehrer im Tausch für spirituellen Rat und rituelle Dienste. In ihrem Hoheitsgebiet unterstützten sie das Wirken ihrer Präzeptoren und die Gründung monastischer Niederlassungen und begünstigten dabei, daß die buddhistische Lehre sich in der zweiten Phase ihrer Ausbreitung auf regionaler Ebene entwickelte und eine enorme Diversität verschiedener Lehrsysteme und Ritualpraktiken hervorbrachte. Insbesondere im Raum Zentraltibets (Ütsang) des 14. und 15. Jahrhunderts läßt sich ein starker Anstieg religiöser Bautätigkeit, Klostergründungen, scholastischer Produktivität und eine Neuorientierung der verschiedenen Schulrichtungen des Buddhismus feststellen.

Um die geschichtlichen Ereignisse der Region sowie vorherrschende Wert- und Glaubensvorstellungen jener Zeit besser zu verstehen, scheint es unausweichlich, das Mönchstum in die religionsgeschichtliche Untersuchung des »tibetischen Mittelalters" miteinzuschließen. Erkenntnisse über jene dominierende Gemeinschaftsform ordinierter Buddhisten können dazu beitragen, gegenwärtige Entwicklungen in der tibetischen Gesellschaft neu zu bewerten und die stark idealisierte und politisch funktionalisierte Tibetwahrnehmung Außenstehender geradezurücken.
Eine Auseinandersetzung mit dem Klosterwesen vor der Einrichtung der Ganden Photrang- oder Dalai Lama-Regierung im Jahr 1642 hat in der Tibetforschung bisweilen nur partiell stattgefunden. Ein Großteil der urkundlichen Zeugnisse und historischen Dokumente, welche direkten Einblick in Klosterstrukturen und Organisation geben könnten, ist aufgrund der einschneidenden politischen Veränderungen in der Mitte des 20. Jahrhunderts verschollen oder der westlichen Forschung nicht zugänglich. Monastisch-religiöse Literatur hingegen ist in ihrem Wesen häufig so beschaffen, daß sie nur Teilbereiche einer sozio-historischen Untersuchung bedienen kann und daher eine gesicherte Rekonstruktion der Lebens- und Vorstellungswelt der Mönche nicht immer ermöglicht. Eine vielversprechende Quellengattung für die Erforschung der monastischen Lebenswelt vor Einführung der Dalai Lama Administration bildet die biographische Literatur aus Tibets Klöstern.

\section{Lebensgeschichten als "befreites Wirken" (namthar)}

Der tibetischen Leserschaft sind biographische Texte, die das Leben religiöser Leitfiguren beschreiben, unter dem Gattungstitel namthar oder nampa tharpa vertraut. Sie erzählen zumeist das Leben historisch verbürgter Personen, welche als spirituelle Lehrer respektvoll mit dem Titel Lama (Sanskrit: guru) oder rimpoche verehrt werden. Die portraitierten Akteure sind buddhistische Lehrmeister und Tantriker, inkarnierte Linienhalter, Mönchsgelehrte, inthronisierte Klostervorsteher, Wanderasketen und Eremiten.

Namthar, die geläufigste Textbezeichnung für solche Lebensdarstellungen, be-
Ganden Songtsenling Gompa, Kreis ShangriLa, Autonomer Bezirk der Tibeter Dêqên, Provinz Yunnan. Bild: Wikimedia commons deutet wörtlich »vollkommene Befreiung« und bezeichnet das Heilsziel im Buddhismus: die Erlangung der Befreiung aus dem Daseinskreislaufs (Sanskrit: samsara) von Geburt und Tod.

Nach tibetischer Glaubensauffassung sind jene spirituellen Lehrer oder Lamas, deren vorbildliches Leben in den narrativen Texten geschildert ist, vom Leid des Daseinskreislaufs befreit und haben bereits den Zustand der allwissenden Buddhaschaft in einem vorhergehenden Leben verwirklicht. In seiner gegenwärtigen Existenz wird der Lama beziehungsweise Lehrmeister als jemand verstanden, welcher zwar das Heilsziel erlangt und vollkommenen erwacht ist, anstatt aber in einen nirvanisch, befriedeten Zustand einzukehren, in menschlicher Form in die leidvolle Welt hinabsteigt, um den unwissenden, unbefreiten Lebewesen die "Gute Lehre« aufzuzeigen und sie zur eigenen Befreiung zu führen. So heißt es in der namthar des Tantrameisters Gongkar Kunga Namgyal (1432-1496):

Auch wenn [Du] den unveränderlichen $\mathrm{Zu}$ stand [der Buddhaschaft] verwirklicht hast, bringst [Du] die Wesen auf den Pfad von Woblergehen und Glïck,

durch [Dein] unermeßliches [Heils]wirken [unterschiedlicher] Erscheinungsformen

in den endlos, vielen [Daseins]bereichen. ${ }^{4}$

Namthar ist aber nicht allein die genrespezifische Bezeichnung für diese Art von biographischer Literatur. Es meint in seiner wörtlichen Bedeutung vor allem das aus der »Befreiung « resultierende Heilswirken und die spirituellen Wundertaten eines Meisters, welcher nach allgemeiner Auffassung von Mitgefühl für die »fühlenden Wesen« getrieben zu ihrem Wohl tätig wird. Deren Lebens- 
darstellungen als biographische Erzählungen eines exemplarischen Weges hin zur Befreiung verstehen zu wollen, scheint nicht ganz treffend. Glaubt man den Verfassern, reproduzieren sie mit der namthar lediglich Ausschnitte aus dem nicht faßbaren Heilswirken ihrer bereits erwachten Protagonisten, die sich lediglich in spiritueller Schulung präsentieren. Im Kontext der biographischen und historischen Geschehnisse haben die religiös verstandenen Texte aber nicht alleine das Wirken des gegenwärtigen Lebens zum Gegenstand. Sie behandeln oft auch die Vorleben ihrer verwirklichten Akteure, die in Gestalt namhafter Meister und Asketen schon in der Vergangenheit zum Wohl der Lebewesen tätig waren. Die Lehre von vielfältigen Erscheinungsformen der Buddhas und die Vorstellung von spirituellen Meistern als deren menschliche Verkörperung (tulku) haben sich in Tibet dahingehend entwickelt, daß aus ihnen ein tatsächliches Inkarnationssystem von wiederkehrenden Meistern und Linienhaltern wie der Dalai Lama-Linie hervorgegangen ist.

Für das namthar-Genre, eine Textform, welche also das befreite Wirken ihres Protagonisten schildert, hat es sich etabliert, von Hagiographien zu sprechen, auch wenn es in Tibet weder Heilige noch Heiligsprechung im eigentlichen Sinne gibt. Die namentliche Adaption einer ursprünglich christlichen Gattungsbezeichnung liegt dabei in der Vergleichbarkeit hinsichtlich des literarischen Stils und der religiösen Funktion. Wie ihr abendländisches Gegenstück bedient sich die tibetische Hagiographie einer Reihe religiös-verwurzelter Glaubensmodelle, Lehrinhalte und Wertvorstellungen, um das geläuterte Leben ihres Protagonisten mit entsprechender Rhetorik zu veranschaulichen. Die Einbeziehung kanonischer Textauszüge, lokaler Narrative und Analogien zum Buddhaleben und den Heilstaten tibetischer und indischer Meister sind dabei gern verwendete Stilmittel in einem Genre, welches Prosatext und lyrische Anteile gleichermaBen aufzuweisen weiß. In der Beschreibung vorbildlicher "Tugenden" und spiritueller "Errungenschaften" seines Hauptakteurs stützt sich das namthar auf gängige Stereotype, religiöse Symbolik und indisch-buddhistische Erklärungsmodelle. Idealtypisch werden das heilspendende Handeln und die vermeintlich spirituelle Entwicklung des Lamas schemenhaft im Lichte des buddhistischen Erlösungsweges dargestellt, ohne indes den geschichtlichen Kontext oder biographische Eigenheiten völlig auszublenden. ${ }^{5}$

In ihrer Funktion gleichen namthar-Texte christlichen Heiligenviten insofern, als sie am Beispiel der vorbildlichen Lebensführung und Gesinnung ihrer Akteure inspirieren und belehren möchten.

Eine Hauptfunktion der tibetischen Vitaliteratur liegt explizit darin, Hingabe zum Meister und zur buddhistischen Lehre im Geiste seiner Leser hervorzurufen. Für den Rezipientenkreis, der monastischen Gemeinschaft, wird spirituelle Verwirklichung am Beispiel der individuellen Lebensgeschichte erfahrbar und bekommt eine reale Dimension. Ferner wird der buddhistischen Lehre kraft jener Texte Gültigkeit im Hinblick auf ihre Wirksamkeit, Praktikabilität und das Heilsversprechen in der Gesamtheit nachgewiesen. Die klassischen Lebensbeschreibungen des Buddha und Lebensgeschichten prominenter Mönchsgelehrter, Lehrmeister und Wanderasketen werden, wenn auch nicht Teil des monastischen Lehrplans, noch heute als heils- und segensbringende Lektüre in den Klöstern gelesen.

Belehrend und instruktiv können tibetische Hagiographien verstanden werden, indem sie theoretische Lehrinhalte und Glaubensvorstellungen am lebensgeschichtlichen Beispiel greifbar machen und dem Leser wesentliche Stationen, Übungen und Hindernisse auf dem buddhistischen Pfad im Kontext der individuellen Biographie und historischen Gegebenheiten verdeutlicht werden. Ein nicht geringer Anteil der Erzählung widmet sich hierbei der religiösen Schulung und dem spirituellen Werdegang der Akteure unter Anleitung bewanderter Lehrer. In einem hierarchisch, stark von Imitation geprägten Studien- und Lehrsystem wie dem des traditionellen Tibet ist es nicht verwunderlich, daß vorgelebte Sittlichkeit und Einsicht schon allein als eine Form religiöser Belehrung aufgefaßt werden.

Wie das Abfassen religiöser Texte im allgemeinen hat auch das Verschriftlichen von Lebensdarstellungen prominenter Meister das Ziel, den Leser zu belehren und ihn seiner eigenen Befreiung näherzubringen. So schließt der Autorenkolophon einer Hagiographie gewöhnlich mit der Widmung, daß diese Lebensdarstellung des Meisters die unverzügliche Befreiung aller fühlenden Wesen und den zukünftigen Erhalt der buddhistischen Lehre bedingen möge.

In verkürzter, lyrischer Form haben Hagiographien vermutlich Anwendung als Inspirationsquelle und Inventar für Lobpreise und Bittgebete gefunden. Mit dem Einzug hagiographischer Elemente in die liturgische Literatur behalten der Lama und sein altruistisches Wirken als Gegenstand zeremonieller Gebete und täglicher Rezitation ihre Lebendigkeit innerhalb der Mönchsgemeinde.
Rechts: The Grand Monastery of Tashi-Lhunpo, aus: Sarat Chandra Das, Journey to Lhasa and Central Tibet, 1902.

Des weiteren haben Autoren religionsgeschichtlicher Werke fleißig aus den Hagiographien einzelner Meister geschöpft und diese zu großen Teilen übernommen. Religions- und Klostergeschichten, Abt- oder Inkarnationschronologien, Genealogien und Annalen sind zumeist Sammlungen von Einzelbiographien, die lediglich verkürzt wurden. Die Zahl der eigenständigen Biographien übersteigt dabei deutlich die Zahl historiographischer Werke.

Obgleich Hagiographien innerhalb der religiösen Literatur Tibets nur einen geringen Anteil einnehmen, ist das namthar ein äußerst beliebtes und weitverbreitetes Textgenre von vielfältiger Anwendung und eine für Historiker, Anthropologen und Religionswissenschaftler wesentliche Quelle aus dem klösterlichen Milieu. Als Blockdruck haben sich hagiographische Schriften seit dem frühen 15. Jahrhundert weit über den Wirkungsraum einzelner Lamas verbreitet. Umgearbeitet und gekürzt sind sie nicht nur in andere Textgattungen eingeflossen, sondern haben sich in den bedeutenden Vitensammlungen und religionsgeschichtlichen Werken verschiedener Lehrtraditionen über ganz Tibet reproduziert. Untersuchungen zur Textproduktion und -rezeption hagiographischer Literatur haben in der Tibetforschung jüngst Anlauf genommen.

\section{Tibetische Meister (lama)}

Die Popularität und das weite Anwendungsfeld des Genres lassen sich vor allem mit dem eminenten Status von Lamas und religiösen Akteuren im tibetischen Kulturraum erklären. Durch seine höchste spirituelle Verwirklichung und einflußnehmenden Heilsaktivitäten in der Gegenwart wird ihm als Ritualmeister eine elementare Rolle zuteil. Mittels seines Wissens um tantrische Rituale und die religiöse Wahrheit werden ihm übernatürliche Fähigkeiten und absolute spirituelle Autorität zugeschrieben.

Im tibetischen Buddhismus, einer Form des Vajrayana, wie es sich auf beiden Seiten des Himalayas etabliert hat, sind das Studium von Schriften und die Ausführung ritueller Praktiken und kontemplativer Übungen an die Initiation und Anleitung eines ausge- 


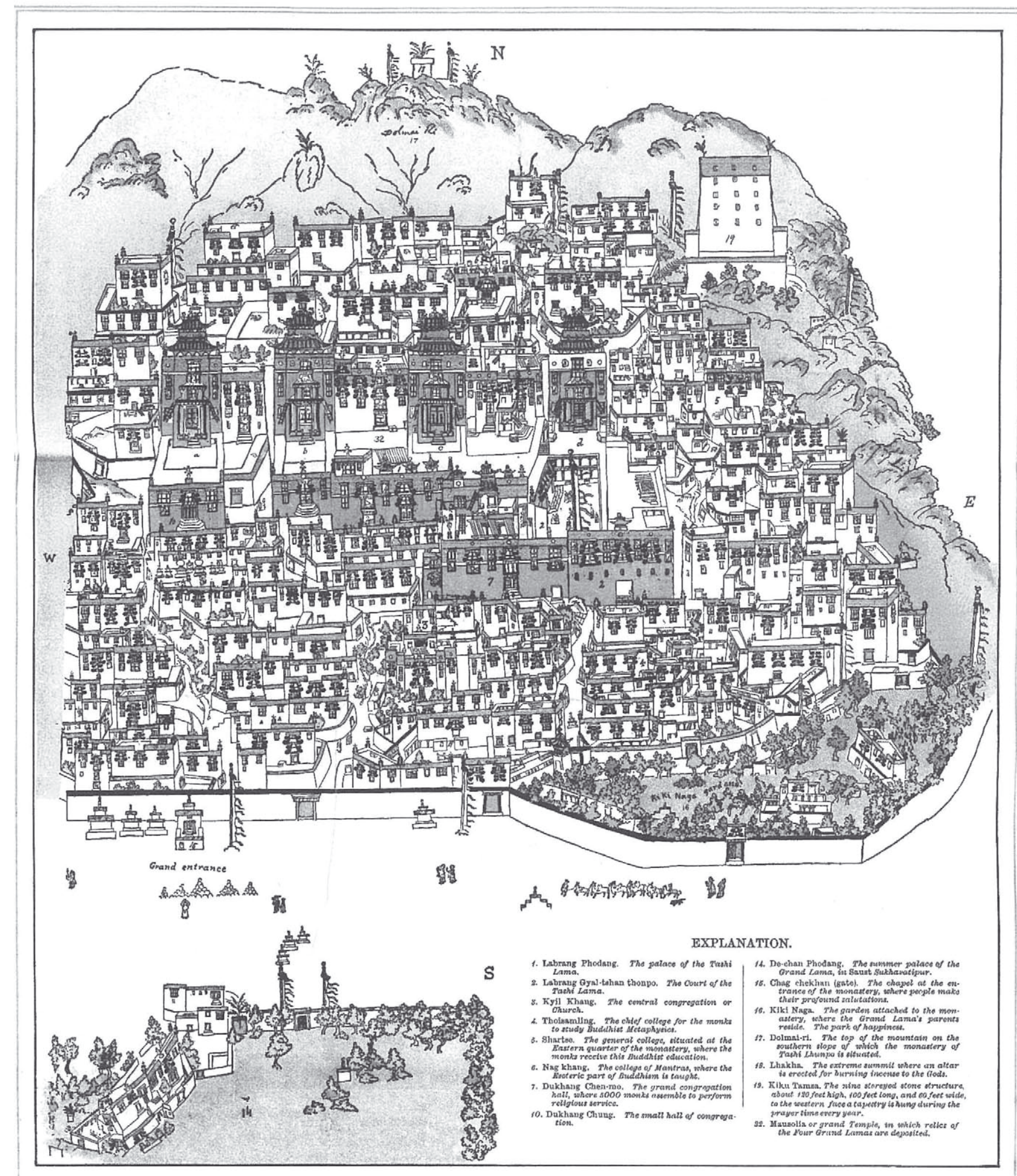

wiesenen Lehrers gebunden. Erst mit der formellen Leseübertragung eines Textes und durch die rituelle Einweihung in Gegenwart des tantrischen Meisters, der seinerseits die Instruktionen im Rahmen einer Überlieferungslinie von seinem Lehrer erhalten hat, ist der Schüler befähigt, esoterische Schriften zu lesen und die assoziierte Praxis auszuführen. Nicht nur ist die praktische Ausübung und intellektuelle Auseinandersetzung mit Lehrtexten ohne formelle Autorisierung unzulässig, nach tibetischer Glaubensauffassung wird dem Text per se ohne diese seine soteriologische Wirksamkeit abgesprochen. Lernen und monastische Schulung organisiert sich demnach um den tantrischen Lehrmeister, dem die Weitergabe von religiösem Wissen vorbehalten ist und durch dessen
Güte erst spiritueller Fortschritt des Einzelnen möglich wird.

Im Besitz spirituell erworbener Kräfte und als Mensch gewordener Buddha steht der Lama im Zentrum des religiösen Universums seiner Gemeinschaft, in seiner Wertigkeit nicht verschieden vom Religionsstifter Siddhartha Gautama. Diese unübertreffliche Autorität des Lamas, die sich in der 


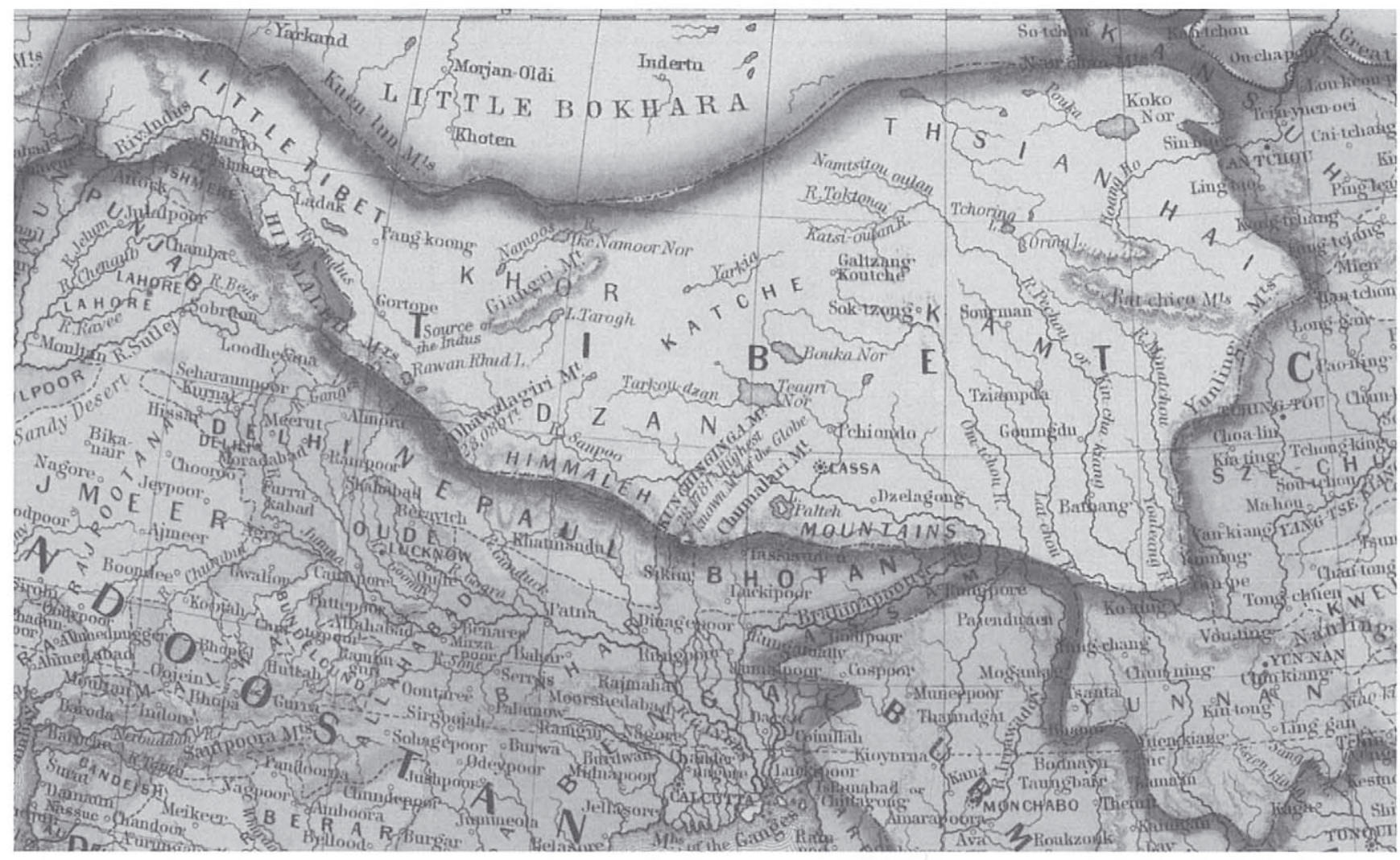

Wortbedeutung seines Titels erhalten hat, findet Ausdruck in vielen Aspekten der monastischen Alltagskultur und der devotionalen Haltung seitens der Laienänhanger. Von seiner Schülerschaft, der Klostergemeinde und Laienbevölkerung, wird er als maßgeblicher Vermittler von Spiritualität wahrgenommen, welcher mittels ritueller Einflußnahme imstande ist, auf weltliche Zusammenhänge und übernatürliche Phänomene und Wesenheiten einzuwirken. Nach volkstümlichem Verständnis sind es diese rituellen Befähigungen, die als Voraussetzung für sein erfolgreiches Wirken und die Verbreitung der buddhistischen Heilslehre zu betrachten sind.

Der spirituell bemühte, gewöhnliche Mönch hingegen stellt als Teil der Schülerschaft an deren Spitze der Lama steht, den Großteil der monastischen Gemeinde. Er schult sich innerhalb der klösterlichen Gemeinde unter Anleitung in den ihm zugewiesenen Tätigkeitsbereichen wie Schriftauslegung, Ritualpraxis und Kontemplation. Mönche genießen gesellschaftliche Anerkennung als religiöse Spezialisten, welche dem häuslichen Leben entsagt haben und nach buddhistischen Idealen leben.

\section{Spurensuche}

Es waren zumeist gelehrte Mönche, welche die ersten Lebensdarstellungen nach dem Ableben ihrer Meister verfaßten. Aus den
Autorenkolophonen wird ersichtlich, daß es oftmals ein begnadeter Schüler und persönlicher Bediensteter des Meisters war, welcher wiederholt um die Niederschrift der Lebensgeschichte von einem Bittsteller ersucht und von Lokalherrschern mit den nötigen Mitteln ausgestattet wurde. Ist der Verfasser nicht selten im unmittelbaren Kreis der Schüler zu finden, so scheint es die weitere Schülerschaft des Meisters zu sein, welche der Autor als primären Rezipientenkreis versteht. Dennoch schließt die Kompilation weiterreichende Ambitionen des Autors nicht aus, den Meister und sein Lehrsystem über die Anhängerschaft hinaus im religiösen Diskurs zu verorten und ihm einen zeitlosen Platz im Kreis der spirituell Verwirklichten und Buddhas zu sichern.

Über den monastischen Lehrbetrieb ist bekannt, daß die Lebensgeschichten in formeller Textübertragung innerhalb der Klöster tradiert worden sind. So machen es Hagiographien zukünftigen Generationen von Mönchen möglich, sich in Beziehung zu bringen mit zentralen Persönlichkeiten und den Gründervätern ihrer Tradition, welche durch Reinkarnation immer wieder in die monastische Gemeinschaft zurückzukehren vermögen. In diesem Sinne bilden die Texte nicht nur die Vorstellungswelt des ihr entsprungenen Milieus $a b$, sondern tragen gleichzeitig zum Selbstverständnis der Gruppe bei. Die Bedeutungsdimension ha- giographischer Literatur für die Gemeinschaft kann nur schwer überbewertet werden, und es ist die im tantrischen Buddhismus zugewiesene Rolle von Lehrmeistern, welche die Akteure gleichzeitig mit einer gemeinschaftsbildenden Funktion ausgestattet hat. Insbesondere für die Zeit der Späten Verbreitung, in der sich der Buddhismus im Wirkungskreis spiritueller Lehrer und dessen Förderer weithin regional entfaltete und eine Vielfalt von Strömungen herausbildete, wird der gesellschaftliche Einfluß und Stellenwert religiöser Leitfiguren deutlich.

Der Einfluß des Lamas geht dabei weit über das Kloster selbst hinaus. Im regionalen Umfeld war er zugleich eine politische Gestalt, die in Zusammenarbeit mit dem Gabenherrn ein politisches Territorium definierte - als den Geltungsbereich seiner spirituellen Macht. Dem religiösen Thron (densa) selbst ist dieser Geltungsbereich gleichsam eingeschrieben. In seinen Funktionen als Klostergründer, Ritualmeister, Lehrverkünder und Mediator in gemeinschaftlichen Konflikten bezieht das immer auch die Laienbevölkerung in den Kreis seiner Gemeinschaft mit ein. In den Gründungsgeschichten von religiösen Institutionen, wie sie in den Oraltraditionen mitunter bis heute fragmentarisch noch erhalten sind, lassen sich oft der weitreichende Einfluß und die profane Macht des Lamas und seines Thrones gut erkennen. ${ }^{6}$ 
Links: Tibet (Ausschnitt aus: Hindoostan, Farther India, China, and Tibet, Mitchell's new general atlas, containing maps of the various countries of the world, plans of cities, etc., embraced in fifty-three quarto maps., forming a series of eighty four maps and plans, together with valuable statistical tables. (In 2 layers). 1860. Quelle: The Lionel Pincus \& Princess Firyal Map Division, The New York Public Library. - Rechts: Der Meister Gongkar Kunga Namgyal (1432-1496) als Miniatur abgebildet in der über ihn verfaßten Biographie (Anm. 5) Folio 1a, rechts.

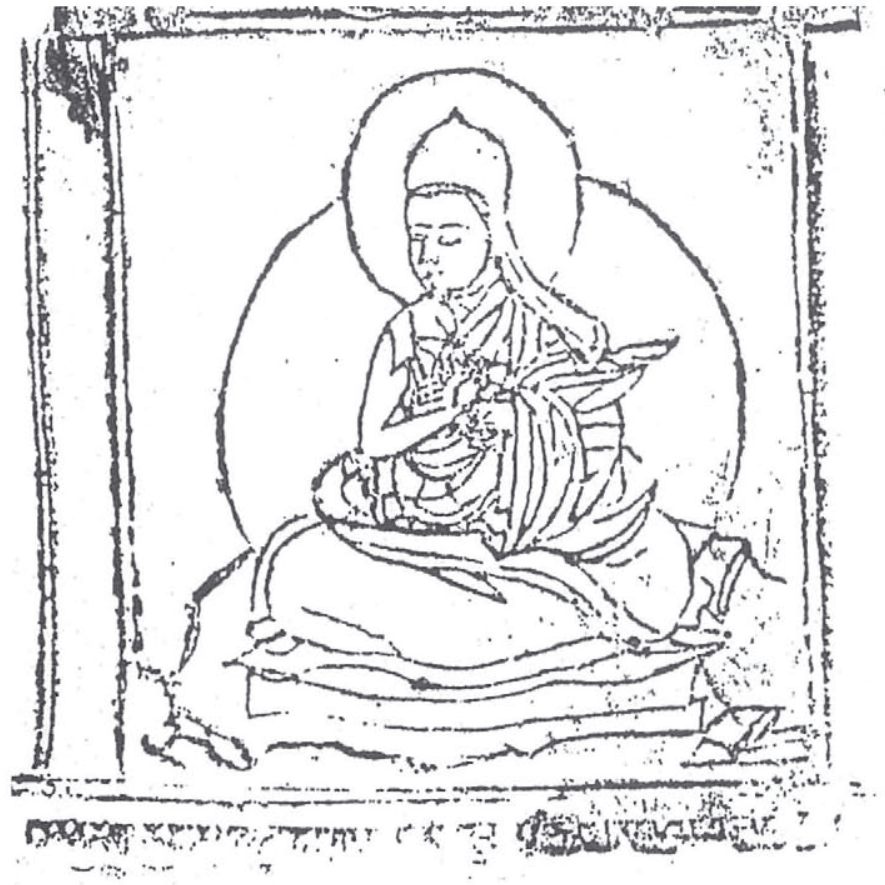

Einfluß von Täuschung und im Gebrauch weltlicher Konventionen zu erfassen möglich ist. ${ }^{7}$ das religiöse Umfeld ihrer Protagonisten, vorherrschende Gemeinschaftsvorstellungen, die soziale Lebenswelt monastischer Gruppen, aber auch über die Mechanismen der Integration der Lokalbevölkerung bringen. Ungeachtet des idealtypischen Charakters und des Verzichtes, triviale Gegebenheiten einzufangen, lassen sich mit Hilfe von Lebensgeschichten Rückschlüsse auf die im Erzählhintergrund befindlichen Klöster ziehen. Ihr Gründungsmythos und Aufbau wie auch monastische Praktiken und Festivitäten sind fester thematischer Bestandteil des hagiographischen Schreibens. Die Einbeziehung ergänzender Quellentexte und anthropologischer Datenerhebungen aus Feldforschung macht es unter anderem möglich, Interaktionszonen der Klöster herauszuarbeiten, historische Ereignisse im monastischen Kontext zu bemessen und Toponyme der religiösen Landschaft geographisch zu verorten.

Für den Autor einer namthar hingegen leiten sich der sozio-religiöse, historische und geographische Erzählrahmen ganz aus der Gegenwart und dem Handeln des Protagonisten ab. Was er mittels der namthar mitzuteilen vermag, ist seiner Auffassung nach lediglich das Heilswirken eines Buddha gleichen Meisters, wie es ihm und den anderen "gewöhnlichen«, unbefreiten Wesen unter

\section{Anmerkungen}

* Die Darstellung basiert auf vorläufigen Ergebnissen des laufenden Dissertationsprojekts über die Formation monastischer Gemeinschaften der Sakya Lehrtradition im Raum des südlichen Zentraltibet zur Zeit der Phagmodrupa Herrschaft (1354-ca. 1480). Als Textgrundlage dienten dementsprechend lebensgeschichtliche Werke von Sakya-Meistern der Region. Univ.-Doz. Dr. Guntram Hazod (Institut für Sozialanthropologie, Österreichische Akademie der Wissenschaften, Wien) danke ich für Anmerkungen zu inhaltlichen Aspekten dieses Beitrags.

1. Einleitender Vers zur Abfassungsintention der Biographie von Jamyang Dragpä Pel, alias rDzong pa Kun dga' rgyal mtshan (13831446). dPal ldan chos skyong, Chos rje rdzong pa kun dga' rgyal mtshan pa'i rnam thar; Dehradun 2008 (?), 1: sangs rgyas kun dngos 'jam dbyangs grags pa'i dpall / rdo rje sems dpa'i go 'phang mobog brnyes kyang / /'gro ba'i don du yongs 'dzin tshul bstan pa'il / rnam thar mdor bsdus bdag gis brjod par byal

2. Dung dkar Blo bzang 'phrin las, Bod kyi chos srid zung 'brel skor bshad pa, Peking 1981,106.

3. Melvyn Goldstein, Tibetan Buddhism and Mass Monasticism [Englische Übersetzung des französischen Beitrags erschienen in: Adeline Herrou/Gisele Krauskopff (Hg.), Des moines et des moniales dans le monde. La vie monastique dans le miroir de la parenté, Toulouse 2010], 3. Online verfügbar auf den Netzseiten des Center For Research on Tibet, Case Western Reserve University: http://www.case.edu/affil/tibet/ tibetanMonks/documents/Tibetan_Bud dhism_and_Mass_Monasticism.doc (abgerufen am 03.02.2014)

4. rGya ston Byang chub dbang rgyal, rD zong pa kenn dga' rnam rgyal gyi rnam thar, Lhasa 2001 , 13: 'pho med go 'phang mngon gyur kyang / / zhing khams rab 'byams mtha'yas par/ / sprulpa'i mdzad pa grangs med kyis/ / 'gro rnams phan bde 'i lam la bleod/

5. Ein brauchbarer Überblick über die Textgattung der tibetischen Biographie findet sich in Giuseppe Tucci, Tibetan Painted Scrolls, Rom 1949, Band 1, 150-170.

6. Vgl. hierzu die Fallstudie von Tsering Gyalbo/ Guntram Hazod/Per K. Sørensen, Civilization at the Foot of Mount Sham-po: The Royal House of IHa-Bug-pa-can and the History of g.Ya'-bzang; Historical Texts from the Monastery of $g . Y a^{\prime}-b z a n g$ in Yar-stod (Central Tibet). Annotated Translation, Transliteration and Facsimile Edition, Wien 2000.

7. Nach buddhistischer Erklärung ergibt sich dieses relative Wirklichkeitsverständnis der Welt aus geistiger Verblendung, wechselseitiger $\mathrm{Ab}$ hängigkeit der Dinge und weltlichen Konventionen. Siehe Candrakīrti (ca. 7. Jahrhundert n. Chr.), Prasannapadā, 492,10-12. Dazu auch Helmut Tauscher, Die Lehre von den zwei Wirklichkeiten in Tson kha pas Madbyamaka-Werken, Wien 1995, 199 f., 215-218.

Mathias Fermer, Tibetan Studies, Institut für Kultur- und Geistesgeschichte Asiens, Österreichische Akademie der Wissenschaften, Apostelgasse 23,1030 Wien, mathias.fermer@oeaw.ac.at 


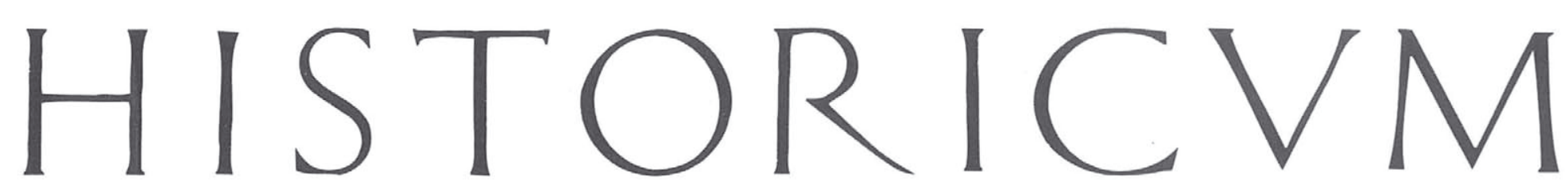

ZEITSCHRIF T UUR GESCHICHTE

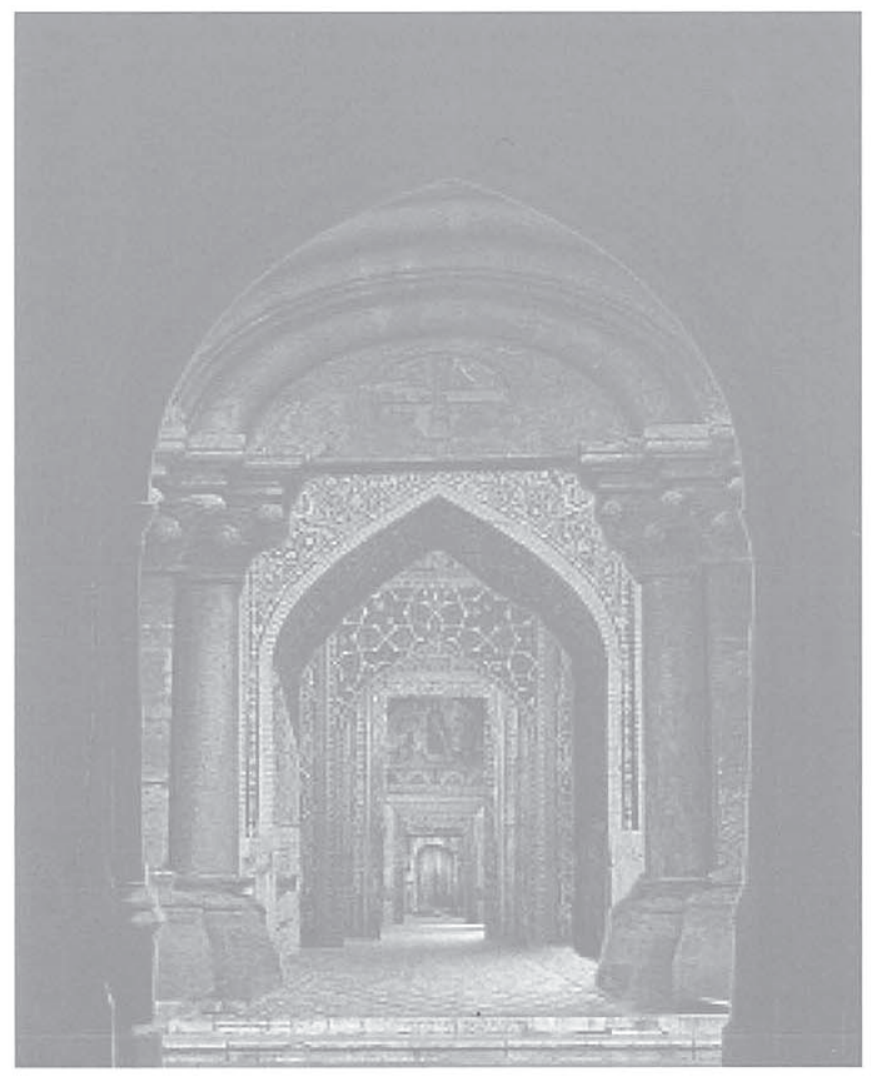

SOMMER - HERBST 20012

VISIONS OF COMMUNITY

$H A B$ I LI T A T I O N

D I R K K R U P P N O W 


\section{Editorial}

Das vorliegende Heft präsentiert Beiträge aus einem interdisziplinären Sonderforschungsbereich des Fonds zur Förderung der wissenschaftlichen Forschung: Mittelalterliche Gemeinschaftsvorstellungen in christlichen, islamischen und buddhistischen Gesellschaften werden dabei vergleichend untersucht. Angesiedelt ist das Projekt an der Österreichischen Akademie der Wissenschaften und an der Universität Wien.

Die Beiträge des Heftes stammen aus einer Ringvorlesung an der Universität Wien, die Gemeinschaftsvorstellungen jeweils mit exklusivem Blick auf eine Gemeinschaft behandelt hat. Der Umfang dieser Gemeinschaften variiert freilich stark und reicht vom christlichen Europa insgesamt bis zu einer einzelnen dalmatinischen Insel. Inkludiert sind Südarabien und Tibet ebenso wie Österreich und das Karolingerreich.

Die Autoren arbeiten überwiegend an

Titelbild: Tore. Corporate Image des Sonderforschungsbereichs "Visions of Community". verschiedenen Instituten der Akademie der Wissenschaften und der Universität Wien: An der Akademie forschen Gerda Heydemann, Veronika Wieser und Rutger Kramer sind (Institut für Mittelalterforschung), Eirik
Hoven und Daniel Mahoney (Institut für Sozialanthropologie) sowie Mathias Fermer (Institut für Kultur- und Geistesgeschichte Asiens, Bereich Tibetologie). An der Universität Wien arbeiten Christian Opitz (Institut für Kunstgeschichte), Elisabeth Gruber (Institut für Geschichte) sowie Fabian Kümmeler und Sascha Attia (Institut für Osteuropäische Geschichte). Irene van Renswoude ist am Institut für kulturwissenschaftliche Forschung der Universität Utrecht im Bereich der Mittelalterforschung tätig.

Dic Teilprojekte von Visions of Community werden von Walter Pohl, Andre Gringrich, Helmut Krasser (von den genannten Instituten der ÖAW) sowie Christina Lutter und Oliver Schmitt (von den Instituten der Universität Wien) geleitet. Rutger Kramer, einer der Autoren dieses Heftes, ist auch Koordinator zwischen den Teilprojekten.

Eine Einführung ins Konzept des Sonderforschungsbereiches gibt die Einleitung von Walter Pohl (Gesamtleiter) zu Beginn dieses Schwerpunkts.

Michael Pammer

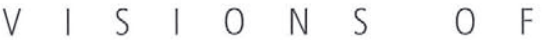 \\ Visions of Community< im interkulturellen Vergleich Von Walter Pohl \\ Gemeinschaftsvorstellungen in Zeiten

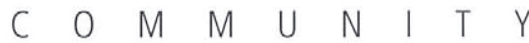 \\ Bilder von Gemeinschaften - Bilder für Gemeinschaften: Zur visuellen Kultur spätmittelalterlicher Dominikanerklöster in Mitteleuropa Von Christian Nikolaus Opitz des Umbruchs: Der Gebrauch der Bibel in Europa zwischen Antike und Frühmittelalter Von Gerda Heydemann und Veronika Wieser

Medieninhaber und Verleger: Aktionsgemeinschaft/ÖSU.Herausgeber:Michael Pammer. Sitz des Verlagesundder Redaktion: Finkengang 27, A4048 Linz-Puchenau. Hersteller: Salzkammergut Media Ges. m. b. H., 4810 Gmunden. Erscheinungsort: Linz. Verlagspostamt: 4040 Linz. Telephon $+43 / 664 / 60246-7000$ Fax $+43 / 732 / 2468-8532$

hup: / |www.wsg-bist.uni-linz.ac.at| Historicum. bom

E-Mail:historicum@jku.at

Konto: PSK (BLZ 60000) 1026.722/HISTORICUM

HISTORICUM dient der Diskussion von Fragen det Geschichtswissenschaft und der Politik. Namentlich gekennzeichnete Beiträge müssen nicht mit der Meinung der Redaktion übereinstimmen.
Dissens, Debatte und Diskurs: Kirche und Imperium in der Karolingerzeit Von Rutger Kramer und Irene van Renswoude

Stammes- und Religionsgemeinschaften im mittelalterlichen Südarabien Von Eirik Hovden und Daniel Mahoney

Tibetische Meister und ihr »befreites Wirken«: Auf Spurensuche monastischer Gemeinschaften in den Lebensgeschichten des mittelalterlichen Tibet Von Mathias Fermer
Städtische Gemeinschaftsbildung im spätmittelalterlichen Herzogtum Österreich

14 Von Elisabeth Gruber

Der Strafe zum Trotz: Gemeinschaft und Konflikt im venezianischen Dalmatien: Ein Blick auf Korčula im 15. Jahrhundert Von Fabian Kümmeler und Sascha Attia

$\begin{array}{llllllllllll}H & A & B & \text { I } & \text { L } & \text { I } & \text { T } & \text { A } & \text { T } & \text { I } & 0 & \mathrm{~N}\end{array}$

28 Dirk Rupnow: 》Judenforschung« im Dritten Reich Von Michael Pammer

Impressum

Ausstellungen 